\title{
Lessons from an Australian community dialectical behaviour therapy programme for borderline personality disorder
}

\section{Catherine Thompson}

Specialist Registrar, Adult Psychiatry, North West Shropshire Community Mental Health Trust, email cathmjthompson@hotmail.com

$\mathrm{n}$ 2003, the National Institute for Mental Health in England (NIMHE) published guidelines for the development of services for people with personality disorders (NIMHE, 2003), prompting community mental health teams (CMHTs) to reassess their service provision for this patient group. The guidelines did not recommend any particular treatment approach, but CMHTs were encouraged to develop specialist programmes. For many CMHTs the focus has been on borderline personality disorder, as it is one of the most prevalent personality disorders seen in adult mental health services and has high costs for both the patient and the service (Moran, 2002). The evidence base for which treatment may be most effective within the community remains small, however, and this has left many $\mathrm{CMHTs}$ unclear regarding a suitable programme. Here I will outline a CMHT-based dialectical behaviour therapy (DBT) programme operating in Western Australia and reflect on why I feel it is an option that CMHTs in the UK looking to develop their service should consider.

Dialectical behaviour therapy is just one of the emerging treatments with evidence for its effectiveness in borderline personality disorder. It was developed by Marsha Linnehan specifically to address the needs of patients with this disorder (Linnehan, 1993a,b). The model combines both cognitive and behavioural therapies within a framework of understanding borderline personality disorder; the dialectic in this therapy is between validation and acceptance of patients as they are, while at the same time encouraging change in behaviours and emotional regulation. Palmer (2002) provides further details on the components of DBT.

\section{The model in action}

Peel Community Mental Health serves a mixed rural and suburban population of approximately 92000 within Western Australia (Peel Development Commission, 2005). It comprises both a general adult and a separate child and adolescent mental health service (CAMHS). It employs an equivalent of 30 multidisciplinary clinicians, including occupational therapists. The population of the area is rapidly growing, but has a relatively low socio-economic profile and a lack of community infrastructure associated with the development of a new region. The epidemiology of disorders, service provision, workload and skill base of clinicians are comparable to those seen in my experience of UK services.

The DBT model was selected by Peel Community Mental Health because, at the time of implementation (2000), it was felt to be among the best researched, and the manualised approach allowed for ease of training within the community setting. The Peel service did not receive funding for the development of the programme and DBT was felt to be cheaper than other programmes. Also, most of the clinicians had some experience of cognitive-behavioural therapy and felt more comfortable with learning the related DBT skills than with psychodynamic techniques.

The programme at Peel is essentially true to Linnehan's original model, although with a few alterations to account for the practical constraints of a CMHT. The programme was initially devised to be an early intervention and was targeted at those aged 15-25 years (i.e. incorporating some CAMHS patients); it has since broadened to include all ages. People with comorbid diagnoses are accepted to the programme but these conditions should not dominate the clinical picture.

Patients with borderline personality disorder identified at assessment are encouraged to engage with the DBT programme. A contract is agreed with each patient for psychotherapy for at least 1 year and a maximum of 3 years, with an undertaking to attend a skills group for an hour once a week. A primary therapist is allocated from within the multidisciplinary team, who agrees to provide regular individual psychotherapy, on average once every 2 weeks, and to coordinate psychosocial rehabilitation. Cases are divided equally among the team in order to ensure a balanced caseload, and a maximum case-load of three is recommended. In-patient admissions are encouraged to be brief and planned; any necessary crisis admissions should be less than 3 days, with in-patient staff agreeing not to engage in therapy and any changes in medication to be made in collaboration with the out-patient team. Management with adjunctive pharmacological agents is encouraged to be via primary care, and general practitioners have been educated with regard to the presentation patterns of borderline personality disorder and the nature of the programme in place at Peel.

More complex cases and those with comorbidity may be managed pharmacologically by psychiatrists within the service, who need not act as the primary therapist. Contrary to Linnehan's model, out-of-hours telephone contact is not provided by the primary therapist and instead all patients presenting in situational crisis are referred to the duty worker if the therapist is not available; this decision was made principally on the practical grounds of CMHT workers not being able to provide a 24-hour service for patients. The duty officer is trained to manage crises as appropriate, but not to intervene in therapy.

A weekly peer supervision group is held, in which the skills group facilitators feed back on the progress of the group in 
the previous session. Therapists offer support to each other and collaborate in management strategies.

\section{Personal perspective}

Before working at Peel, my experience of managing patients with borderline personality disorder had been generally negative: I often felt ill-equipped to deal with their behaviour and I was conscious that my management was not always consistent. My perceived lack of skill fuelled countertransference towards them.

I do not believe that DBT represents the gold standard in treatment for borderline personality disorder, but it has given me a framework from which to work and the results I have seen are positive. I was surprised at how quickly I was able to learn the theory of DBT and, like some other clinicians at Peel, I have received only brief training in the basic theory of DBT. However, I believe that with the support of more experienced peers, clinicians are able to draw on their previous mental health experience to offer the validation required in individual therapy and reinforce skills learnt in the group; this makes DBT a practical option for CMHTs, which can, with a little extra training, utilise a skill base already in place.

I had previously worked in CMHTs with no specific treatment programme, where lack of structure and guidance on how to manage borderline personality disorder frequently resulted in splitting within the team. I recall lengthy hospital admissions being used for want of any alternative. I have also worked in CMHTs which have had access to specialist programmes, one run on a psychodynamic basis and another based on a DBT model. I was not directly involved in either programme and cannot comment on the efficacy of the therapy itself, but I do recall a sense of detachment of these services from the CMHT. There was a tendency for clinicians immediately to refer patients with borderline personality disorder to these services, without thinking of a management plan themselves; if the patient were then to present in crisis to the CMHT, management could turn to panic and often resort to hospital admission. The holistic and multidisciplinary approach of a DBT programme based within, and run by, a
CMHT ensures all clinicians are exposed to the often demanding cases of borderline personality disorder and yet no group is overwhelmed by them. It also ensures that no clinician becomes deskilled in the management of such patients and provides the patients with vital continuity of care.

I have also been able to apply these new skills to other patients, such as those with eating disorders and substance misuse problems.

The peer group supervision was invaluable in gaining support and provided an opportunity to interact with other disciplines, one which I have often found lacking in other CMHTs.

The clinicians at Peel were keen to innovate and develop services; their sense of pride in this programme was tangible and well deserved. Perhaps it is this team approach which, at least in part, transfers to and benefits the patient group. Bearing in mind those treatments that have been shown to be effective, the determination of which of these to employ may be less important than the team developing a common approach with a sense of purpose, which in itself leads to successful treatment. I believe that DBT allows for such an approach and my time at Peel inspired me with hope that even a small service with relatively few resources and little extra funding can provide an effective treatment option for borderline personality disorder. CMHTs in the UK looking to develop their service would do well to consider a similar approach.

\section{References}

Linnehan, M. M. (1993a) Cognitive Behavioural Treatment of Borderline Personality Disorder. Guildford.

Linnehan, M. M. (1993b) Skills Training Manual for Treating Borderline Personality Disorder. Guildford.

Moran, P. (2002) The epidemiology of personality disorder. Psychiatry, 1, 8-11.

NIMHE (2003) Personality Disorder: No Longer a Diagnosis of Exclusion. Policy Implementation Guidance for the Development of Services for People with Personality Disorders. NIMHE.

Palmer, R. L. (2002) Dialectical behaviour therapy for borderline personality disorder. Advances in Psychiatric Treatment, 8, 10-16.

Peel Development Commission (2005) Peel Development Commission Annual Report 2004-05. Government of Western Australia.

\section{The yield from national surveys of mental health}

\section{Scott Henderson FRCPsych ${ }^{1}$ and Gavin Andrews FRCPsych ${ }^{2}$}

'Emeritus Professor, John Curtin School of Medical Research, Australian National University, Canberra, ACT 0200, Australia, email ashenderson@netspace.net.au

${ }^{2}$ Scientia Professor, School of Psychiatry, UNSW at St Vincent's Hospital, Sydney, NSW, 2010, Australia

\begin{abstract}
t least 21 countries have now carried out national surveys of mental health under the aegis of the World Health Organization's World Mental Health Surveys. This has meant interviewing some 157000 people in their homes. The countries are as varied as Australia, China,
\end{abstract}

Iran, six continental European nations, Nigeria, the UK and the USA (Andrews et al, 2001; Demyttenaere et al, 2004; Mohammadi et al, 2005). It is therefore timely to consider what this very large body of information has yielded and to what use it can be put, especially in relation to the costs 